\title{
Bijective proofs of the hook formulas for the number of standard Young tableaux, ordinary and shifted
}

\author{
C. KrattenthaleR ${ }^{\dagger}$ \\ Institut für Mathematik der Universität Wien, \\ Strudlhofgasse 4, A-1090 Wien, Austria.
}

Submitted: February 20, 1995; Accepted: July 9, 1995

\begin{abstract}
Bijective proofs of the hook formulas for the number of ordinary standard Young tableaux and for the number of shifted standard Young tableaux are given. They are formulated in a uniform manner, and in fact prove $q$-analogues of the ordinary and shifted hook formulas. The proofs proceed by combining the ordinary, respectively shifted, Hillman-Grassl algorithm and Stanley's $(P, \omega)$-partition theorem with the involution principle of Garsia and Milne.
\end{abstract}

1. Introduction. A few years ago there had been a lot of interest in finding a bijective proof of Frame, Robinson and Thrall's [1] hook formula for the number of standard Young tableaux of a given shape. This resulted in the discovery of three different such proofs $[2,10,14]$, none of them is considered to be really satisfactory. Closest to being satisfactory is probably the proof by Franzblau and Zeilberger [2]. However, while the description of their algorithm is fairly simple, it is rather difficult to show that it really works. Also, it does not portray the nice row-column symmetry of the hooks. Remmel's proof [10] is the most complicated. It uses the involution principle of Garsia and Milne [3]. However, Remmel bases his proof on "bijectivization" of recurrence relations, which is not the most direct route to attack the problem. Finally, Zeilberger's proof [14], translating the beautiful probabilistic proof [6] by Greene, Nijenhuis and Wilf into a bijection, actually sets up a bijection between larger sets than one desires.

So, it is still considered to be the case that the best proof of the hook formula is to use the Hillman-Grassl algorithm [7] and Stanley's $(P, \omega)$-partition theorem [12], and then to apply a limit argument (this is the non-bijective part). In view of this it is somehow surprising that there are half-combinatorial proofs of the hook formula

1991 Mathematics Subject Classification. Primary 05A15; Secondary 05A17, 05A30, 05E10, 05E15, $11 \mathrm{P} 81$.

Key words and phrases. Standard Young tableaux, shifted standard tableaux, hook formula, Hillman-Grassl algorithm, $(P, \omega)$-partition theorem, involution principle.

†Supported in part by EC's Human Capital and Mobility Program, grant CHRX-CT93-0400 and the Austrian Science Foundation FWF, grant P10191-PHY 
that were translated into bijective proofs, such as the Gessel-Viennot method [4, 5] of nonintersecting lattice paths in Remmel's proof [10], and the probabilistic proof [6] by Greene, Nijenhuis and Wilf in Zeilberger's proof [14], but that it was never tried to translate what is still considered to be the best (but only half-combinatorial) proof into a bijective proof. This omission is made up for in this paper. In fact, it turns out to be pretty simple. Besides, this new bijective proof has two advantages. First, it actually proves a natural $q$-analogue of the hook formula (see the Theorem in section 4) which all the other proofs do not. Secondly, the same idea also works to provide a bijective proof of the hook formula for the number of shifted standard Young tableaux (and its $q$-analogue; see the Theorem in section 4). No bijective proof for the shifted hook formula has been given before. On the other hand, our proofs are still not satisfactory in that they involve the involution principle.

The outline of the paper is as follows. In the next section we review all relevant definitions. Then, in section 3, we explain briefly what the Hillman-Grassl algorithm, Stanley's $(P, \omega)$-partition theorem and the involution principle of Garsia and Milne are about. Finally, in section 4 we state the two hook formulas and present our bijective proofs of them, in a unified fashion. In section 5 we explain where the involutions of section 4 come from.

2. Definitions. A partition of a positive integer $n$ is a sequence $\lambda=\left(\lambda_{1}, \lambda_{2}, \ldots\right.$, $\left.\lambda_{r}\right)$ with $\lambda_{1}+\lambda_{2}+\cdots+\lambda_{r}=n$ and $\lambda_{1} \geq \lambda_{2} \geq \cdots \geq \lambda_{r}>0$, for some $r$. The components of $\lambda$ are called the parts of $\lambda$. The integer $n$, the sum of all the parts of $\lambda$, is called the norm of $\lambda$ and is denoted by $n(\lambda)$. The (ordinary) Ferrers diagram of $\lambda$ is an array of cells with $r$ left-justified rows and $\lambda_{i}$ cells in row $i$. Figure 1.a shows the Ferrers diagram corresponding to $(4,3,3,1)$. If $\lambda$ is a partition with distinct parts then the shifted Ferrers diagram of $\lambda$ is an array of cells with $r$ rows, each row indented by one cell to the right with respect to the previous row, and $\lambda_{i}$ cells in row $i$. Figure 1.b shows the shifted Ferrers diagram corresponding to $(5,4,2,1)$. We shall frequently use the same symbols for things which may have an "ordinary" or "shifted" interpretation. It will always be clear which interpretation is meant. In particular, if a partition $\lambda$ appears in the shifted context then it is always assumed that $\lambda$ is a partition with distinct parts.

The conjugate of a partition $\lambda$ is the partition $\left(\lambda_{1}^{\prime}, \ldots, \lambda_{\lambda_{1}}^{\prime}\right)$ where $\lambda_{j}^{\prime}$ is the length of the $j$-th column in the ordinary Ferrers diagram of $\lambda$.

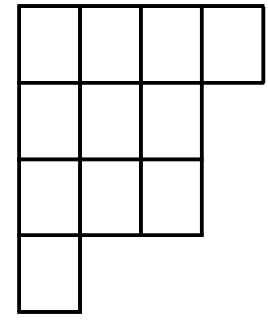

a. Ferrers diagram

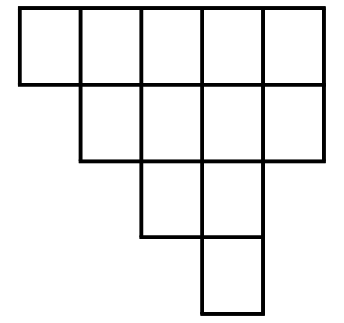

b. shifted Ferrers diagram

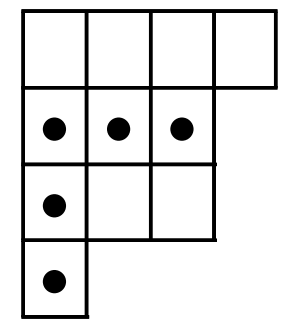

c. hook

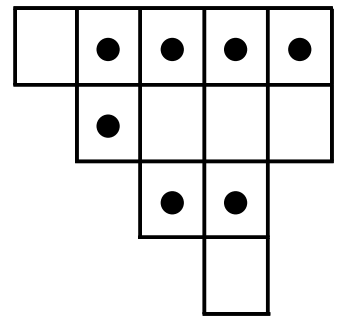

d. shifted hook

Figure 1 
We label the cell in the $i$-th row and $j$-th column of the ordinary, respectively shifted, Ferrers diagram of $\lambda$ by the pair $(i, j)$. Also, if we write $\rho \in \lambda$ we mean ' $\rho$ is a cell of $\lambda^{\prime}$. The hook of a cell $\rho$ of the ordinary Ferrers diagram of $\lambda$ is the set of cells that are either in the same row as $\rho$ and to the right of $\rho$, or in the same column as $\rho$ and below $\rho, \rho$ included. The dots in Figure 1.c indicate the hook of the cell $(2,1)$. The hook of a cell $\rho$ of the shifted Ferrers diagram of $\lambda$ again includes all cells that are either in the same row as $\rho$ and to the right of $\rho$, or in the same column as $\rho$ and below $\rho, \rho$ included, but if this set contains a cell on the main diagonal, cell $(j, j)$ say, then also all the cells of the $(j+1)$-st row belong to the hook of $\lambda$. The dots in Figure 1.d indicate the hook of the cell $(1,2)$. The hook length $h_{\rho}$ (in the ordinary, respectively shifted sense) of a cell $\rho$ of $\lambda$ is the number of cells in the hook of $\rho$.

\begin{tabular}{|l|l|l|l|}
\hline 1 & 3 & 4 & 4 \\
\hline 5 & 5 & 7 & \multicolumn{1}{|l}{} \\
\cline { 1 - 2 } 5 & 6 & 7 & \multicolumn{1}{|c}{} \\
\cline { 1 - 2 } 8 & \multicolumn{3}{|c}{} \\
\cline { 1 - 2 } & & &
\end{tabular}

a. reverse plane partition

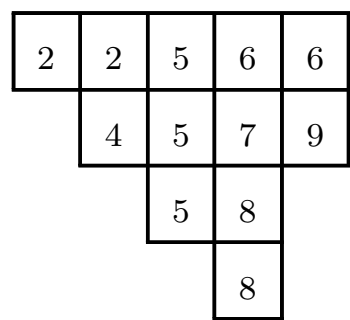

b. shifted reverse plane partition

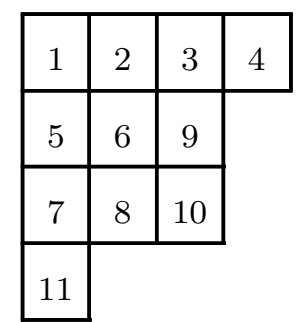

c. standard Young tableau

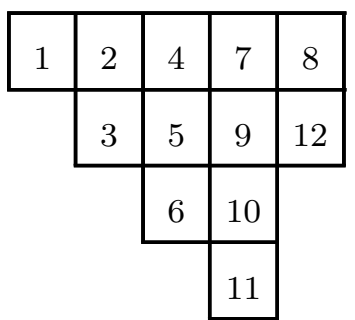

d. shifted standard Young tableau

Figure 2

Given a partition $\lambda=\left(\lambda_{1}, \lambda_{2}, \ldots, \lambda_{r}\right)$, a reverse plane partition of shape $\lambda$ (in the ordinary or shifted sense) is a filling $P$ of the cells of $\lambda$ with nonnegative integers such that the entries along rows and along columns are weakly increasing. Figure 2.a displays an ordinary reverse plane partition of shape $(4,3,3,1)$, Figure 2.b displays a shifted reverse plane partition of shape $(5,4,2,1)$. We write $P_{\rho}$ for the entry in cell $\rho$ of $P$. Also here, we call the sum of all the entries of a reverse plane partition $P$ the norm of $P$, and denote it by $n(P)$. Given a partition $\lambda$ of $n$, a standard Young tableau of shape $\lambda$ (in the ordinary or shifted sense) is a reverse plane partition whose set of entries is $\{1,2, \ldots, n\}$. Figure 2.c displays an ordinary standard Young tableau of shape $(4,3,3,1)$, Figure 2.d displays a shifted standard Young tableau of shape $(5,4,2,1)$.

\begin{tabular}{|l|l|l|l|}
\hline 11 & 10 & 9 & 8 \\
\hline 7 & 6 & 5 & \multicolumn{1}{|c}{} \\
\cline { 1 - 2 } 4 & 3 & 2 & \multicolumn{2}{|c}{} \\
\cline { 1 - 2 } 1 & \multicolumn{2}{|c}{} \\
\cline { 1 - 2 } & & \multicolumn{2}{|c}{}
\end{tabular}

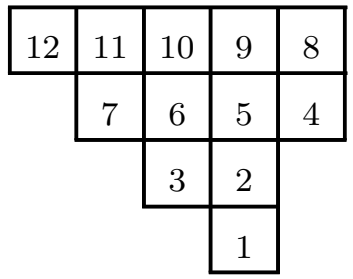

Figure 3

For the rest of the paper we fix the following total order of the cells of an (ordinary or shifted) Ferrers diagram $\lambda$. A cell $\rho_{1}$ comes before cell $\rho_{2}$ if $\rho_{1}$ is in a lower row 
than $\rho_{2}$ or if both are in the same row but $\rho_{1}$ is to the right of $\rho_{2}$. In other words, to obtain the total order one starts with the right-most cell in the last row and reads each row from right to left, beginning with the bottom row and continuing up to the first row. We write $\# \rho$ for the number of the cell $\rho$ in this total order. Figure 3 displays the values $\# \rho$ for the ordinary diagram $(4,3,3,1)$ and the shifted diagram $(5,4,2,1)$.

Next we define a statistics on (ordinary or shifted) standard Young tableaux, which is similar to charge (see [8; 9, p. 129] for the definition of charge). Given a standard Young tableau $Y$ with $n$ entries, we define $\operatorname{comaj}(Y)$ to be the sum $\sum(n-i)$, where the sum is over all $i$ that have the property that $i+1$ is located strictly above $i$ in $Y$. For example, comaj(.) $=3$ for the standard Young tableau in Figure 2.c and $\operatorname{comaj}()=.9+6+1=16$ for that in Figure 2.d.

We call an arbitrary filling of the cells of $\lambda$ (ordinary or shifted) with nonnegative integers a tabloid of shape $\lambda$. Also here, by $n(T)$ we mean the sum of all the entries of $T$, and by $T_{\rho}$ we mean the entry in cell $\rho$ of $T$. Furthermore, we define the hook weight $w_{h}(T)$ of a tabloid $T$ of shape $\lambda$ by $\sum_{\rho \in \lambda} T_{\rho} \cdot h_{\rho}$, where $h_{\rho}$ has to be understood in the ordinary or shifted sense, depending on whether the shape $\lambda$ is understood in the ordinary or shifted sense. Finally, we introduce some special tabloids to be used in the course of the following bijections. We call a tabloid $T$ of shape $\lambda$ a $(<h)$-tabloid if $T_{\rho}<h_{\rho}$ for all cells $\rho \in \lambda$, and we call $T$ a $(0-h)$-tabloid if $T_{\rho}$ equals 0 or $h_{\rho}$, for all cells $\rho \in \lambda$. Similarly, we call a tabloid $T$ of shape $\lambda$ a $(<\#)$-tabloid if $T_{\rho}<\# \rho$ for all cells $\rho \in \lambda$, and we call $T$ a $(0-\#)$-tabloid if $T_{\rho}$ equals 0 or $\# \rho$, for all cells $\rho \in \lambda$. The $\operatorname{sign} \operatorname{sgn}(T)$ of a $(0-h)$-tabloid or $(0-\#)$-tabloid is always defined to be $(-1)$ number of nonzero entries in $T$.

3. Preliminaries. In this section we briefly explain the basic ingredients of our bijections in the next section: the ordinary and shifted Hillman-Grassl algorithm, a bijection that comes from Stanley's $(P, \omega)$-partition theory, and the involution principle of Garsia and Milne.

Let $\lambda$ be a partition of $n$. The ordinary Hillman-Grassl algorithm [7] sets up a bijection, HG say, between reverse plane partitions $P$ of (ordinary) shape $\lambda$ and tabloids $S=\mathrm{HG}(P)$ of (ordinary) shape $\lambda$, such that

$$
n(P)=w_{h}(S)
$$

where the hook weight $w_{h}$ is read in the ordinary sense. Sagan's shifted HillmanGrassl algorithm [11, sec. 3,4] does the same for reverse plane partitions of shifted shape $\lambda$ and tabloids of shifted shape $\lambda$, provided that the hook weight $w_{h}$ in $(3.1)$ is now read in the shifted sense.

The second bijection that we need is a bijection, SP say, between reverse plane partitions $P$ of shape $\lambda$ (ordinary, respectively shifted) and pairs $(Y, \tau)$, where $Y$ is a standard Young tableau of shape $\lambda$ (ordinary, respectively shifted), and $\tau$ is a partition with at most $n$ parts. It comes from $[12$, sec. 6]. Given the reverse plane partition $P$, the standard Young tableaux $Y$ is given by the numbering of the cells of $\lambda$ that is determined by reading the entries of $P$ according to size, starting with the smallest entry and going up to the largest entry, if two entries are equal the one 
in the higher row comes first, and if two equal entries are in the same row, the left entry comes first. See the examples below. The partition $\tau$ is formed in the following way. Consider the entries of $P$ in the order just described. At the very beginning (i.e. when considering the entry in cell $(1,1)), 0$ is subtracted from the considered entry. Suppose that we subtracted $s$ from the entry considered last. Then we subtract $s$ from the next entry to be considered if it is located weakly below the previously considered entry, otherwise we subtract $s+1$. The partition $\tau$ is the sequence of all the obtained integers, in reverse order, and disregarding all 0's. For example, under this mapping the reverse plane partition in Figure 2.a is mapped to (Figure 2.c, 76665554431), and the reverse plane partition in Figure 2.b is mapped to (Figure 2.d,666544444422). It is not difficult to check that this correspondence satisfies

$$
n(P)=n(\tau)+\operatorname{comaj}(Y) .
$$

Finally we recall the involution principle of Garsia and Milne [3] (see also [13, sec. 4.6]). Let $X$ be a finite set with a signed weight function $w$ defined on it. Furthermore, let $X_{L}$ and $X_{R}$ be subsets of $X$, both of which containing elements with positive sign only. Suppose that there is a sign-reversing and weight-preserving involution $i_{L}$ on $X$ that fixes $X_{L}$ and a sign-reversing and weight-preserving involution $i_{R}$ on $X$ that fixes $X_{R}$. Then there must be a weight-preserving bijection between $X_{L}$ and $X_{R}$. And such a bijection can be constructed explicitly by mapping $x \in X_{L}$ to $\left(i_{L} \circ i_{R}\right)^{m}(x)$ where $m$ is the least integer such that $\left(i_{L} \circ i_{R}\right)^{m}(x)$ is in $X_{R}$.

4. The hook formulas and their bijective proofs. The hook formulas that we are going to prove are the following.

Theorem. Let $\lambda$ be a partition of $n$. Then, in the ordinary or shifted sense, there holds

$$
\sum_{\substack{Y \text { a SYT of } \\ \text { shape } \lambda}} q^{\operatorname{comaj}(Y)}=\frac{[n][n-1] \cdots[1]}{\prod_{\rho \in \lambda}\left[h_{\rho}\right]}
$$

where by definition $[k]:=1+q+q^{2}+\cdots+q^{k-1}$. (SYT is short for 'standard Young tableau'.)

PROOF. Since the formula and all other things that are needed are stated uniformly for the ordinary and shifted case, also the proof can be formulated uniformly, i.e. the following can be read in the ordinary context or in the shifted context.

First we rewrite (4.1) in the form

$$
[n][n-1] \cdots[1]=\left(\sum_{\substack{Y \text { a SYT of } \\ \text { shape } \lambda}} q^{\operatorname{comaj}(Y)}\right) \prod_{\rho \in \lambda}\left[h_{\rho}\right] .
$$

We prove (4.2) by setting up a bijection between the set $O_{L}$ of all $(<\#)$-tabloids $T$ of shape $\lambda$, the generating function $\sum_{T} q^{n(T)}$ for which being evidently the lefthand side in (4.2), and the set $O_{R}$ of pairs $\left(Y, U^{\prime}\right)$, where $Y$ is a standard Young 
tableau of shape $\lambda$ and $U^{\prime}$ is a $(<h)$-tabloid of shape $\lambda$, the generating function $\sum_{\left(Y, U^{\prime}\right)} q^{\text {comaj }(Y)} q^{n\left(U^{\prime}\right)}$ for which being evidently the right-hand side of (4.2). We do this by using the involution principle. Hence, we have to say which choices we take for the set $X$, the signed weight $w$, the subsets $X_{L}$ and $X_{R}$, and the involutions $i_{L}$ and $i_{R}$. Of course, $O_{L}$ and $O_{R}$ should correspond to $X_{L}$ and $X_{R}$, respectively, the latter being subsets of the bigger set $X$, that has to be described next.

We define $X$ to be the set of all triples $(S, T, U)$, where $S$ is an arbitrary tabloid of shape $\lambda$, where $T$ is a $(<\#)$-tabloid of shape $\lambda$, and where $U$ is a $(0-h)$-tabloid of shape $\lambda$. The signed weight $w$ on $X$ is defined by

$$
w((S, T, U))=\operatorname{sgn}(U) q^{w_{h}(S)+n(T)+n(U)} .
$$

We define the set $X_{L}$ to be the subset of $X$ consisting of all triples $(\mathbf{0}, T, \mathbf{0})$, where $\mathbf{0}$ denotes the filling of $\lambda$ with 0 in each cell, and where $T$ is an arbitrary $(<\#)$ tabloid of shape $\lambda$. Note in particular that the sign of $w((\mathbf{0}, T, \mathbf{0}))$ for all these triples is $\operatorname{sgn}(\mathbf{0})=1$, which is positive. Trivially, $X_{L}$ is in bijection with $O_{L}$.

What the set $X_{R}$ is going to be is better explained in the course of the description of the involution $i_{R}$.

However, first we define the involution $i_{L}$ that fixes $X_{L}$. Let $(S, T, U)$ be a triple in $X$ that is not in $X_{L}$, i.e. at least one of $S$ and $U$ is different from $\mathbf{0}$. Pick the least cell $\rho$ (in the total order of the cells defined in section 2) in $\lambda$ such that $S_{\rho} \neq 0$ or $U_{\rho} \neq 0$. If $U_{\rho} \neq 0$, i.e. $U_{\rho}=h_{\rho}$, then replace $U_{\rho}$ by 0 , thus obtaining $\bar{U}$, and add 1 to $S_{\rho}$, thus obtaining $\bar{S}$. If $U_{\rho}=0$ then replace $U_{\rho}$ by $h_{\rho}$, thus obtaining $\bar{U}$, and subtract 1 from $S_{\rho}$, thus obtaining $\bar{S}$. We define $i_{L}((S, T, U))$ to be $(\bar{S}, T, \bar{U})$. By construction we have $w((S, T, U))=-w((\bar{S}, T, \bar{U}))$, as required. It is obvious that $i_{L}$ is an involution on $X \backslash X_{L}$.

Next we define the involution $i_{R}$. As promised before, the definition of the set $X_{R}$ that is fixed by $i_{R}$ will naturally appear in the course of the definition of $i_{R}$.

We partition the set $X$ into two disjoint subsets $X^{1}$ and $X^{2}$. By definition, the set $X^{1}$ consists of all triples $(S, T, U)$ where there exists a cell $\rho$ in $\lambda$ such that

$$
h_{\rho} \leq T_{\rho}+U_{\rho}<\# \rho .
$$

The set $X^{2}$ is defined to be the complement $X \backslash X^{1}$.

Now we define $i_{R}$ on the subset $X^{1}$. Let $(S, T, U)$ be a triple in $X^{1}$, i.e. there exists a cell $\rho$ such that (4.4) is satisfied. We assume that $\rho$ is the least such cell (in the total order explained in section 2). If $U_{\rho}=0$ then we replace $U_{\rho}$ by $h_{\rho}$, thus obtaining $\bar{U}$, and we replace $T_{\rho}$ by $T_{\rho}-h_{\rho}$, thus obtaining $\bar{T}$. If $U_{\rho}=h_{\rho}$ then we replace $U_{\rho}$ by 0 , thus obtaining $\bar{U}$, and we replace $T_{\rho}$ by $T_{\rho}+h_{\rho}$, thus obtaining $\bar{T}$. We define $i_{L}((S, T, U))$ to be $(S, \bar{T}, \bar{U})$. It is obvious that in both cases $(S, \bar{T}, \bar{U})$ is in $X^{1}$ again. Besides, there holds $w((S, T, U))=-w((S, \bar{T}, \bar{U}))$, as required. Clearly, $i_{R}$ thus defined on $X^{1}$ is an involution on $X^{1}$.

Next we consider the set $X^{2}$. Instead of directly working with these triples, it is more convenient to map them in an intermediate step by a sign-preserving and weight-preserving bijection, $\varphi$ say, to another set, $\bar{X}^{2}$ say. By definition, $\bar{X}^{2}$ is the 
set of all quadruples $\left(Y, \pi, T^{\prime}, U^{\prime}\right)$, where $Y$ is a standard Young tableau of shape $\lambda$, $\pi$ is a partition with all its parts being at most $n, T^{\prime}$ is a (0-\#)-tabloid, and where $U^{\prime}$ is a $(<h)$-tabloid. The signed weight on $\bar{X}^{2}$ is defined by

$$
w\left(\left(Y, \pi, T^{\prime}, U^{\prime}\right)\right)=\operatorname{sgn}\left(T^{\prime}\right) q^{\operatorname{comaj}(Y)+n(\pi)+n\left(T^{\prime}\right)+n\left(U^{\prime}\right)} .
$$

The bijection between $X^{2}$ and $\bar{X}^{2}$ is defined in the following way. Let $(S, T, U)$ be an element of $X^{2}$, i.e. for all cells $\rho$ in $\lambda$ the relation (4.4) does not hold. Denote the image of $(S, T, U)$ under the mapping $\varphi$ to be defined by $\left(Y, \pi, T^{\prime}, U^{\prime}\right)$. The pair $(Y, \pi)$ is obtained by applying first the inverse of the Hillman-Grassl algorithm to the tabloid $S$, thus obtaining a reverse plane partition $P$, then applying the map SP explained in section 3 to this reverse plane partition, thus obtaining a pair $(Y, \tau)$ consisting of a standard Young tableau $Y$ and a partition $\tau$ with at most $n$ parts, and finally mapping the partition $\tau$ to its conjugate $\pi$, thus obtaining the pair $(Y, \pi)$ consisting of the standard Young tableau $Y$ and a partition $\pi$ all of whose parts are at most $n$. Because of (3.1) and (3.2) we have

$$
w_{h}(S)=n(P)=\operatorname{comaj}(Y)+n(\tau)=\operatorname{comaj}(Y)+n(\pi) .
$$

Now we turn to the construction of $T^{\prime}$ and $U^{\prime} . T^{\prime}$ and $U^{\prime}$ are obtained by doing the following operation on $T$ and $U$ for each cell $\rho$ in $\lambda$. If $U_{\rho}=0$, then, since (4.4) does not hold, we must have $T_{\rho}<h_{\rho}$. Then we replace $U_{\rho}$ by $T_{\rho}$, and we replace $T_{\rho}$ by 0 . If $U_{\rho}=h_{\rho}$, then we must have $T_{\rho}+h_{\rho}=T_{\rho}+U_{\rho} \geq \# \rho$. Then we replace $U_{\rho}$ by $T_{\rho}+h_{\rho}-\# \rho$, and we replace $T_{\rho}$ by $\# \rho$. It is immediate from the construction and from (4.6) that this mapping from $X^{2}$ to $\bar{X}^{2}$ is weight-preserving and sign-preserving. Besides, because our particular total order of the cells implies $h_{\rho} \leq \# \rho$, the mapping can be inverted, as is easily checked. Hence it is a bijection.

Now, finally, we are able to say what the set $X_{R}$ is. It is defined to be the inverse image (under the mapping $\varphi$ from $X^{2}$ to $\bar{X}^{2}$ just described) of the set of all quadruples $\left(Y, \emptyset, \mathbf{0}, U^{\prime}\right)$, where $Y$ is a standard Young tableau of shape $\lambda, \emptyset$ denotes the empty partition, and where $U^{\prime}$ is a $(<h)$-tabloid. Let us denote the latter subset of $\bar{X}^{2}$ by $\bar{O}_{R}$. Observe that the sign of $w\left(\left(Y, \emptyset, \mathbf{0}, U^{\prime}\right)\right)$ for all quadruples in $\bar{O}_{R}$ is $\operatorname{sgn}(\mathbf{0})=1$, which is positive. Clearly, the set $O_{R}$ of right-hand side objects of (4.2) is in bijection with $\bar{O}_{R}$, and, hence, with $X_{R}$.

So all what remains is to define a sign-reversing and weight-preserving involution, $\bar{i}_{R}$ say, on $\bar{X}^{2}$ that fixes $\bar{O}_{R}$. This is because $i_{R}$ is then defined as it is on $X^{1}$, and on $X^{2}$ by $\varphi^{-1} \circ \bar{i}_{R} \circ \varphi$. Let $\left(Y, \pi, T^{\prime}, U^{\prime}\right)$ be an element of $\bar{X}^{2} \backslash \bar{O}_{R}$, i.e. $\pi$ is a nonempty partition or $T^{\prime}$ is nonzero. Let $i$ be the least (positive) integer such that $i$ occurs as a part in $\pi$ or such that the cell $\rho$ with $\# \rho=i$ has a nonzero entry in $T^{\prime}$. If $T_{\rho}^{\prime}$ is nonzero, i.e. $T_{\rho}^{\prime}=\# \rho=i$, then we replace $T_{\rho}^{\prime}$ by 0 , thus obtaining $\bar{T}^{\prime}$, and we add one part of size $i$ to $\pi$, thus obtaining $\bar{\pi}$. If $T_{\rho}^{\prime}=0$, then we replace $T_{\rho}^{\prime}$ by $i=\# \rho$, thus obtaining $\bar{T}^{\prime}$, and we remove one part of size $i$ from $\pi$, thus obtaining $\bar{\pi}$. We define $\bar{i}_{R}\left(\left(Y, \pi, T^{\prime}, U^{\prime}\right)\right)$ to be $\left(Y, \bar{\pi}, \bar{T}^{\prime}, U^{\prime}\right)$. By construction we have $w\left(\left(Y, \pi, T^{\prime}, U^{\prime}\right)\right)=-w\left(\left(Y, \bar{\pi}, \bar{T}^{\prime}, U^{\prime}\right)\right)$, as required. It is easy to check that $\bar{i}_{R}$ is a sign-reversing and weight-preserving involution on $\bar{X}^{2} \backslash \bar{O}_{R}$.

This completes the bijective proof(s) of the hook formula(s) (4.1). 
5. The algebra behind. Here we explain where the operations $i_{L}, i_{R}, \varphi$ of the previous section come from.

First, it should be observed that the generating function $\sum \operatorname{sgn}(U) q^{w_{h}(S)+n(T)+n(U)}$ for all triples $(S, T, U)$ in $X$ is given by

$$
\prod_{\rho \in \lambda} \frac{1}{1-q^{h_{\rho}}} \prod_{i=1}^{n}[i] \prod_{\rho \in \lambda}\left(1-q^{h_{\rho}}\right) .
$$

Evidently, the involution $i_{L}$ just models combinatorially the cancellation of the two products in (5.1). (What survives after the cancellation is the left-hand side of (4.2).)

Next we may rewrite $(5.1)$ as

$$
\prod_{\rho \in \lambda} \frac{1}{1-q^{h_{\rho}}} \prod_{i=1}^{n}\left(1-q^{i}\right) \prod_{\rho \in \lambda}\left[h_{\rho}\right] .
$$

What the map $i_{R}$ on $X^{1}$ and the subsequent transformation of $(T, U)$ to $\left(T^{\prime}, U^{\prime}\right)$ in the mapping $\varphi$ do, is exactly the combinatorial modelling of the transition from (5.1) to $(5.2)$.

Now, by the Hillman-Grassl algorithm(s) we know that the first product in (5.2) is the generating function $\sum q^{n(P)}$ for all reverse plane partitions $P$ of shape $\lambda$. Moreover, by Stanley's $(P, \omega)$-partition theorem [12, Cor. $5.3+7.2]$ we know that the same generating function can be written as

$$
\frac{\sum_{Y \text { a SYT of shape } \lambda} q^{\operatorname{comaj}(Y)}}{\prod_{i=1}^{n}\left(1-q^{i}\right)} .
$$

Substituting this into (5.2) gives that the generating function $\sum \operatorname{sgn}(U) q^{w_{h}(S)+n(T)+n(U)}$ for all triples $(S, T, U)$ in $X$ can also be written as

$$
\sum_{\substack{Y \text { a SYT } \\ \text { of shape } \lambda}} q^{\operatorname{comaj}(Y)} \prod_{i=1}^{n} \frac{1}{\left(1-q^{i}\right)} \prod_{i=1}^{n}\left(1-q^{i}\right) \prod_{\rho \in \lambda}\left[h_{\rho}\right] .
$$

The transformation of $S$ into $(Y, \pi)$ in the mapping $\varphi$, of course, exactly models combinatorially the transition from (5.2) to (5.4). Finally, it is evident that the map $\bar{i}_{R}$ on $\bar{X}^{2} \backslash \bar{O}_{R}$ just models combinatorially the cancellation of the second and third factor in (5.4). (What survives after the cancellation is the right-hand side of (4.2).)

ACKNOWLEDGEMENT. This work was carried out while the author visited the University of California at San Diego. He thanks the University of California and in particular Adriano Garsia for making this visit possible. Besides, he is indebted to Adriano Garsia for drawing his attention to the problem of finding "nice" combinatorial proofs of hook formulas, and to Jeff Remmel who suggested that the above ideas should also work for the shifted hook formula. 
THE ELECTRONIC JOURNAL OF COMBINATORICs 2 (1995), \#R13

\section{References}

1. J. S. Frame, G. B. Robinson and R. M. Thrall, The hook graphs of the symmetric group, Canad. J. Math. 6 (1954), 316-325.

2. D. S. Franzblau and D. Zeilberger, A bijective proof of the hook-length formula, J. Algorithms 3 (1982), 317-343.

3. A. M. Garsia and S. C. Milne, Method for constructing bijections for classical partition identities, Proc. Nat. Acad. Sci. U.S.A. 78 (1981), 2026-2028.

4. I. M. Gessel and X. Viennot, Binomial determinants, paths, and hook length formulae, Adv. in Math. 58 (1985), 300-321.

5. I. M. Gessel and X. Viennot, Determinants, paths, and plane partitions, preprint.

6. C. Greene, A. Nijenhuis and H. S. Wilf, A probabilistic proof of a formula for the number of Young tableaux of a given shape, Adv. in Math. 31 (1979), 104-109.

7. A. P. Hillman and R. M. Grassl, Reverse plane partitions and tableau hook numbers, J. Combin. Theory Ser. A 21 (1976), 216-221.

8. A. Lascoux and M.-P. Schützenberger, Le monoïde plaxique, Noncommutative structures in algebra and geometric combinatorics (A. de Luca, ed.), Quaderni della Ricerca Scientifica del C. N. R., Roma, 1981, pp. 129-156.

9. I. G. Macdonald, Symmetric Functions and Hall Polynomials, Oxford University Press, New York/London, 1979.

10. J. B. Remmel, Bijective proofs of formulae for the number of standard Young tableaux, Linear and Multilinear Alg. 11 (1982), 45-100.

11. B. E. Sagan, Enumeration of partitions with hooklengths, Europ. J. Combin. 3 (1982), 85-94.

12. R. P. Stanley, Ordered structures and partitions, Mem. Amer. Math. Soc. No. 119, American Mathematical Society, Providence, R. I., 1972.

13. D. Stanton and D. White, Constructive Combinatorics, Undergraduate Texts in Math., Springer-Verlag, New York, Berlin Heidelberg, Tokyo, 1986.

14. D. Zeilberger, A short hook-lengths bijection inspired by the Greene-Nijenhuis-Wilf proof, Discrete Math. 51 (1984), 101-108. 